\title{
Flavor Evaluation of UHT Reconstituted Milk Processed by Direct and Indirect Heat Treatment Using Sensors
}

\author{
Yasumichi Mizota*, Hiroaki Matsui ${ }^{1}$, Michio Ikeda, \\ Nobuo Ichihashi, Keiji Iwatsuki² and Kiyoshi Toko ${ }^{3}$ \\ Food Res. \& Dev. Inst., Morinaga Milk Industry Co., Ltd., \\ 5-1-83, Higashihara, Zama-City 228-8583, Japan \\ ${ }^{1}$ Production Dept., Morinaga Milk Industry Co., Ltd., \\ 5-33-1 Shiba, Minato-ku, Tokyo 108-8384, Japan \\ ${ }^{2}$ Food Sci. \& Tech. Inst., Morinaga Milk Industry Co., Ltd., \\ 5-1-83, Higashihara, Zama-City 228-8583, Japan \\ ${ }^{3}$ Dept. of Electronics, Grad. Sch. of Info. Sci. and Elect. Engin., Kyushu Univ., \\ 744 Motooka, Nishi-ku, Fukuoka 819-0395, Japan
}

(Received April 23, 2008; accepted August 12, 2008)

Key words: taste sensor, odor sensor, sensory evaluation, GC, milk, reconstituted milk, platetype UHT pasteurizer, steam infusion-type UHT pasteurizer

We applied sensor analysis to the evaluation of the flavors of five types of milk, including ultra high temperature (UHT) processed milk and four types of reconstituted milk prepared by combining raw milk, skim milk powder, and butter, and performing different pasteurization methods (indirect heating and direct heating). We conducted a comprehensive evaluation of the flavors by analysis with a taste sensor and an odor sensor, sensory evaluation, and gas-chromatography (GC). We determined the flavor characteristics of each type of milk through sensory evaluation. We found that, to add richness, it was effective to use raw milk and use a plate-type UHT pasteurizer with indirect heating. To give a finish with a plain flavor, it was effective to use a steam infusion-type UHT pasteurizer with direct heating. GC analysis revealed a trend that samples with a flat flavor had a smaller amount of detected volatile compounds. In sensor analysis, examination of the relationships between the results of the odor sensor and taste sensor analyses and the sensory evaluation and GC analysis revealed a correlation between the amount of volatile compounds and the "cooked flavor" with the odor sensor, and a correlation between the "cooked flavor" and "saltiness" with the taste sensor. This suggests the possibility of objectively indicating the flavor characteristics of milk in this experimental system using such sensors.

${ }^{*}$ Corresponding author: e-mail: y_mizota@morinagamilk.co.jp 


\section{Introduction}

Various types of milk products are available on the market, for example, milk, lowfat milk, nonfat milk, composition-controlled milk, processed milk, milk drinks, and so on. Drinks containing these milk products generally use raw milk and processedmilk materials made from raw milk, including skim milk powder, cream, butter, and concentrated skim milk, depending on the desired flavors. It has been reported that the resulting flavors vary with the types of raw materials used, ${ }^{(1)}$ the pasteurization temperature, ${ }^{(2)}$ the pasteurization method, ${ }^{(3)}$ and the homogenization pressure. ${ }^{(4)}$ However, the flavor characteristics resulting from combinations of various types of processed-milk materials and different pasteurization methods have not been clarified.

Sensory evaluation is generally performed to determine the flavor characteristics of food. This approach, however, involves some problems, including the effect of subjectivity on data reproducibility, for instance, individual differences and the physical condition of the panel members, the limited number of samples used in consideration of the load placed on the panel, and so on. Recently, there have been an increasing number of studies on methods of more objectively evaluating flavors using taste sensors that measure on a fixed scale. ${ }^{(5)}$ For example, we have previously reported the application of taste sensors and odor sensors to the flavor evaluation of milk. ${ }^{(6,7)}$

The aim of this study was to apply sensors to evaluate the differences in flavor of reconstituted milk produced by combining various types of milk materials and different pasteurization methods. We conducted a comprehensive evaluation of an experimental model system of ultra high temperature (UHT) processed milk and four types of reconstituted milk prepared with a plate-type UHT pasteurizer with indirect heating (hereinafter identified as "Plate") and a steam-infusion-type UHT pasteurizer with direct heating ("Inf"). The evaluation was performed by sensor analysis, sensory evaluation, and GC analysis. We report the results here.

\section{Materials and Methods}

\subsection{Sample preparation}

As shown in Table 1, five types of samples were prepared for this study, consisting of UHT processed milk and four samples made with combinations of two types of reconstituted milk and two types of pasteurizers. The materials used were raw milk collected in our plant, and skim milk powder and butter manufactured from the same raw milk. The pasteurizers were a plate-type pasteurizer (Plate) and an infusion pasteurizer (Inf). The two types of reconstituted milk were reconstituted milk in which raw milk was not blended, and reconstituted milk in which raw milk was blended at a ratio of $50 \%$ to produce the same composition as that of raw milk (fat 4.0\%, solids not fat (SNF) $8.5 \%$ ). Pasteurization was conducted at $130^{\circ} \mathrm{C}$ for $2 \mathrm{~s}$, followed by homogenization at a pressure of $14 \mathrm{MPa}$ (3.5 MPa at a second stage). Samples were prepared once. They were used for the sensory evaluation, GC analysis, odor sensor analysis, and taste sensor analysis. 
Table 1

Milk and reconstituted milk samples.

\begin{tabular}{cll}
\hline Sample No. & Raw material & UHT Pasteurizer \\
\hline 1 & Raw milk 100\% & Plate-type \\
2 & $\begin{array}{l}\text { Raw milk 50\%, } \\
\text { skim milk powder, }\end{array}$ & Plate-type \\
& butter & \\
& Skim milk powder, & Plate-type \\
& butter & \\
& Raw milk 50\%, & Infusion-type \\
& skim milk powder, & \\
& butter & \\
& Skim milk powder, & Infusion-type \\
& butter & \\
\hline
\end{tabular}

The composition of each sample was $4.0 \%$ fat and $8.5 \%$ solids not fat (SNF).

\subsection{Sensory evaluation}

Sensory evaluation was performed in accordance with the method used by Iwatsuki et al. ${ }^{(2)}$ Researchers in their 20s and 30s belonging to the Research and Development Center of our company had previously received a five-primary-taste distinction test, and 10 males and 10 females (in total 20 panel members) who have a keen sense of taste and habitually drink milk evaluated 13 attributes on a seven-point absolute evaluation scale, with the maximum score being +3 and the minimum score being -3 .

\subsection{GC analysis}

\subsubsection{GC/MS}

Volatile compounds in each milk were extracted by a solid phase micro-extraction (SPME) method and analyzed by GC/MS. The extraction was performed by inserting a 50/30- $\mu \mathrm{m}$-thick StableFlex fiber of divinylbenzene/carboxen/polydimethylsiloxane manufactured by SUPELCO into the headspace of a vial containing $10 \mathrm{ml}$ of a sample and allowing the fiber to stand for $30 \mathrm{~min}$ at room temperature. The resulting extract was injected into the GC. GC was performed using an Agilent 6890 gas chromatograph, and MS was performed using an Agilent 5973 mass spectrometer. The separation column was INNOWax (cross-linked polyethylene glycol; $30 \mathrm{~m}$ long $\times 0.25 \mathrm{~mm}$ inner diameter, film thickness $0.5 \mu \mathrm{m}$ ), the carrier gas was helium, and the oven temperature was increased from 40 to $120^{\circ} \mathrm{C}$ at a rate of $4^{\circ} \mathrm{C} / \mathrm{min}$ and from 120 to $250^{\circ} \mathrm{C}$ at a rate of $6^{\circ} \mathrm{C} /$ min. Compounds were identified by the retention time and by comparing mass spectral data from the samples with those of the Wiley library.

\subsubsection{GC}

GC was performed using an Agilent 6890, in which a SIEVERS 355 sulfur chemiluminescense detector (SCD) was mounted. A DB-1 methyl siloxane separation column $(60 \mathrm{~m}$ long $\times 0.32 \mathrm{~mm}$ inner diameter, and thickness of $5 \mu \mathrm{m})$ was used. Helium 
was adopted as the carrier gas, and the oven temperature was raised from 35 to $260^{\circ}$ $\mathrm{C}$ at a rate of $15^{\circ} \mathrm{C} / \mathrm{min}$. After warming a vial containing a $10 \mathrm{ml}$ sample to $40^{\circ} \mathrm{C}$ and maintaining that temperature for $30 \mathrm{~min}, 5 \mathrm{ml}$ of the headspace was injected into the GC.

\subsection{Odor sensor analysis}

A FOX3000 odor sensor (Alpha M.O.S., France) was used, on which 12 metal oxide sensors made of semiconducting materials were mounted. The odor sensors used are listed in Table 2. A special glass container holding a $4 \mathrm{ml}$ sample was warmed to $37^{\circ} \mathrm{C}$ and that temperature was maintained for $5 \mathrm{~min}$. Then the headspace was fed to the sensor section with pure air at a flow rate of $200 \mathrm{ml} / \mathrm{min}$ for $15 \mathrm{~s}$, and the resistance was measured. The response value was determined using the formula:

response value $=($ maximum resistance - initial resistance $) /$ initial resistance

Measurements were conducted four times for each sample. Of these four measurements, three were analyzed.

\subsection{Taste sensor analysis}

The sensor's electric potential response for each sample was measured in accordance with the method of either $\mathrm{Toko}^{\left({ }^{(8)}\right.}$ or Yamada et al. ${ }^{(9)}$ using a taste sensing system SA402 (Intelligent Sensor Technology, Ltd., Japan) equipped with eight electrodes made of lipid/polymer membranes. Table 3 shows the lipids used for the sensor membranes. The sensor's electric potential response for a sample was calculated as an electric potential relative to the sensor's electric potential response for a reference solution. Measurements were conducted five times for each sample. Of those five measurements, four were analyzed. One lot of milk manufactured by our plant (sterilized at $140^{\circ} \mathrm{C}$ for $2 \mathrm{~s}$ ) was used as the reference solution and a rinse solution.

Table 2

The list of odor sensors.

\begin{tabular}{cl}
\hline Channel & Name of sensor \\
\hline 1 & SY/LG \\
2 & SY/G \\
3 & SY/AA \\
4 & SY/GH \\
5 & SY/GCTL \\
6 & SY/GCT \\
7 & T30/1 \\
8 & P10/1 \\
9 & P10/2 \\
10 & P40/1 \\
11 & T70/2 \\
12 & PA2 \\
\hline
\end{tabular}


Table 3

Lipids used for the sensor membranes.

\begin{tabular}{cl}
\hline Channel & Lipid \\
\hline 1 & Palmitic acid \\
2 & Potassium tetrakis(4-chlorophenyl) borate \\
3 & Phosphoric acid di-n-decyl ester* \\
4 & Phosphoric acid di-n-decyl ester* \\
5 & Tetradodecylammonium bromide** \\
6 & Tetradodecylammonium bromide** \\
7 & Trioctylmethylammonium chloride, \\
& Phosphoric acid di(2-ethylhexyl) \\
8 & Tetradodecylammonium bromide, \\
& 1-tetradecanol \\
\hline
\end{tabular}

*,** Amounts of lipid used are different.

\subsection{Statistical analysis}

Tukey HSD test, principal component analysis (PCA), standard deviation in the response values of the odor sensors and the taste sensors, and correlation analysis were performed using SPSS 13.0J software for Windows. Tukey HSD test was used to investigate significant difference between samples in sensory evaluation and between samples in taste sensor analysis. PCA was performed after standardization. Correlation analysis was performed to examine the relationships among the sensory evaluation, the amount of volatile compounds, and the sensor.

\section{Results}

\subsection{Sensory evaluation}

The results of the sensory evaluation are shown in Fig. 1. The flavors of the milk (Sample 1) and the four types of reconstituted milk (Samples 2, 3, 4, and 5), which were adjusted to have the same fat and SNF content as the milk (Sample 1), exhibited different features. Among the five types of milk, Sample 2 (raw milk 50\%, Plate) showed significantly stronger "milk flavor," "cooked flavor," and "milk taste" compared with Sample 5 (raw milk $0 \%$, Inf), and it had a flavor strong in so-called richness. On the other hand, Sample 5 featured a plain flavor.

Comparison of samples using the same pasteurization method, in other words, comparison of the Plate-pasteurized Samples 2 and 3 (raw milk 0\%, Plate), showed the trend that Sample 2, containing raw milk, had stronger "milk flavor," "cooked flavor," "milk taste," "fattiness," and "body." Comparison of the Inf-pasteurized Samples 4 and 5 also showed the trend that Sample 4, containing raw milk, exhibited similarly stronger attributes. Thus, it was found that the use of raw milk can add richness.

Comparison of Samples 2 and 4 (raw milk 50\%, Inf), with the same blending ratio, showed that Sample 4 was weaker in nine attributes: "milk flavor," "cooked flavor," 


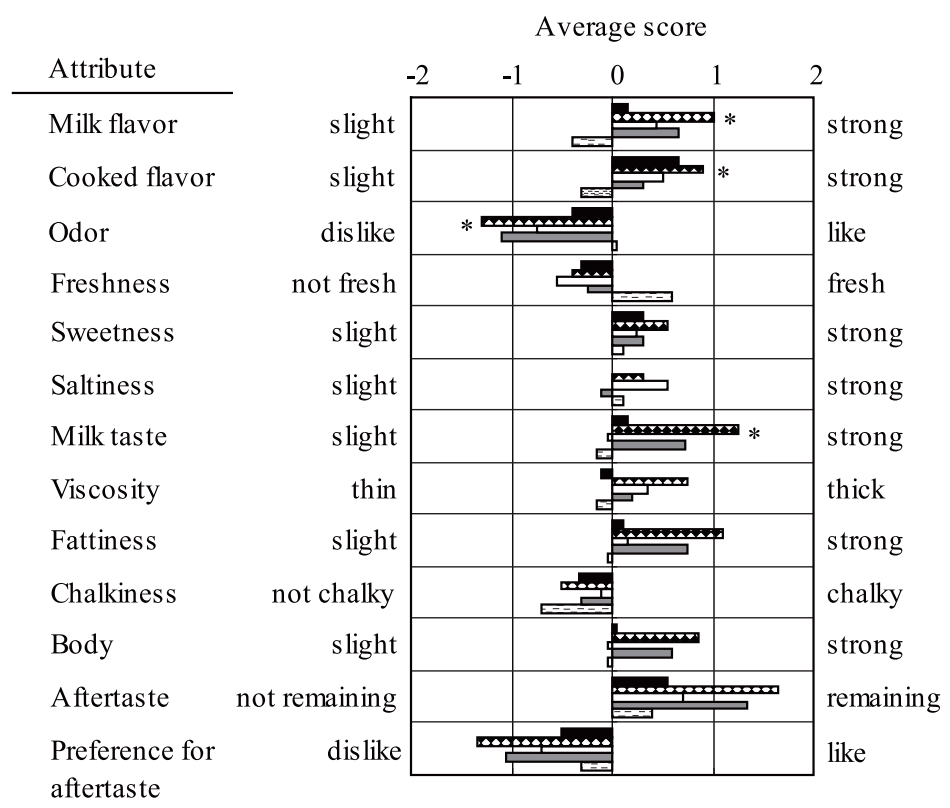

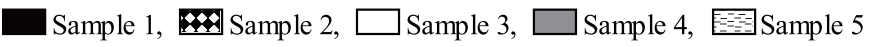

Fig. 1. Average score of the sensory attributes of milk and the four types of reconstituted milk by a panel of experts. *: Significantly different from Sample 5, $p<0.05$.

"sweetness," "saltiness," “milk taste," "viscosity," "fattiness," "body," and "remaining aftertaste." In comparison of Samples 3 and 5, Inf-pasteurized Sample 5 also showed a trend of being similarly weaker. These results are the same as those in a previous study reporting that, with direct heating pasteurization, the effect of the heating was lower, giving a plain flavor. ${ }^{(3)}$

As mentioned, sensory evaluation revealed that the use of different raw materials or pasteurization methods produced different flavor characteristics, even with samples of the same composition, suggesting the possibility of creating desired target flavors.

\subsection{GC and GC/MS analysis}

Figure 2 shows the results of the analysis of five compounds present in large quantities: hexanal, 2-heptanone, 2-nonanone, hydrogen sulfide, and dimethyl sulfide. Compared with the four types of reconstituted milk (Samples 2, 3, 4, and 5), the milk (Sample 1) showed a trend of being abundant in these compounds; in particular, it had a large amount of hydrogen sulfide. In addition, different pasteurization methods produced notable differences; for instance, the Inf-pasteurized Samples 4 and 5 had lower volatile compounds than the Plate-pasteurized Samples 2 and 3.

Comparison of samples using the same pasteurization method, that is, comparison 


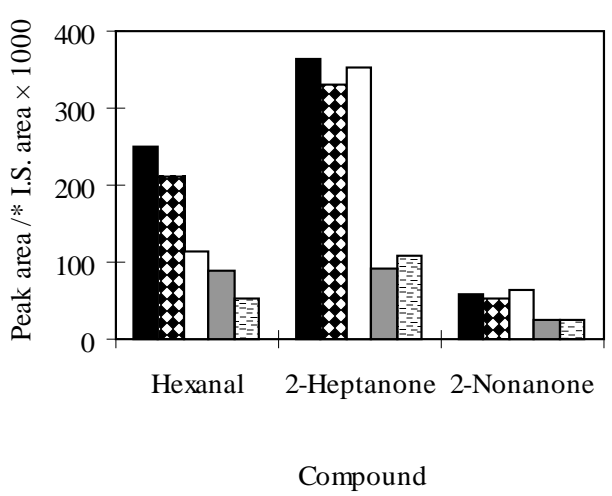

(a)

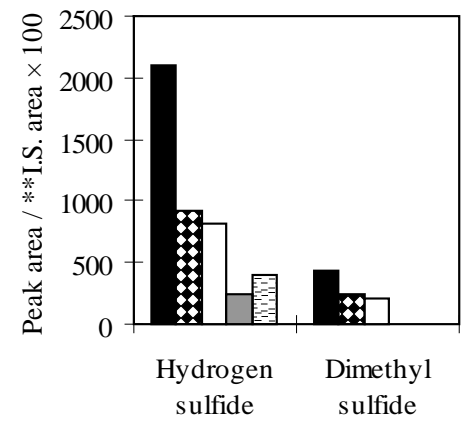

Compound

(b)

Sample 1,

Fig. 2. Volatile compounds in headspace of milk and the four types of reconstituted milk. (a): GC/MS, (b): GC, * I.S.: Ethyl heptanoate, ${ }^{* *}$ I.S.: Ethyl methyl sulfide.

of Samples 2 and 3 and comparison of Samples 4 and 5, did not reveal any marked difference caused by the different blending ratios. Comparison of samples with the same blending ratios, that is, comparison of Samples 2 and 4 and comparison of Samples 3 and 5, revealed that the Inf-pasteurized samples had lower amounts of every compound. These results support the evaluation results of the sensory test described, namely, that the Infpasteurized reconstituted milk has a plain flavor.

\subsection{Odor sensor analysis}

The results of the odor sensor analysis are shown in Fig. 3 and Table 4. Figure 3 shows the results processed by PCA and the following varimax method of the response values of the odor sensors, narrowed down to the three sensors SY/LG, SY/GCTL, and P10/1 by excluding the sensors having high correlations. The proportion of the first principal component (PC1) was $43 \%$, and the proportion of the second principal component (PC2) was $39 \%$, totaling $82 \%$. The results can be substantially accounted for by this two-dimensional diagram. The results were discriminated mainly into a Platepasteurized group and an Inf-pasteurized group. This indicates that the odor sensor analysis discriminated the difference due to the different pasteurization methods, instead of the difference due to the different blending ratios.

Table 4 shows the results of examining the correlation of these odor sensor analysis results with the sensory evaluation and volatile compounds. The results show high correlation of the PC1 in odor sensor analysis with the "cooked flavor" and with every volatile compound, particularly 2-heptanone, 2-nonanone, and dimethyl sulfide. This finding suggests that the $\mathrm{PC} 1$ can be interpreted as an axis indicating the intensity of "cooked flavor" and the amount of volatile compounds. The Plate-pasteurized milk, which had a 


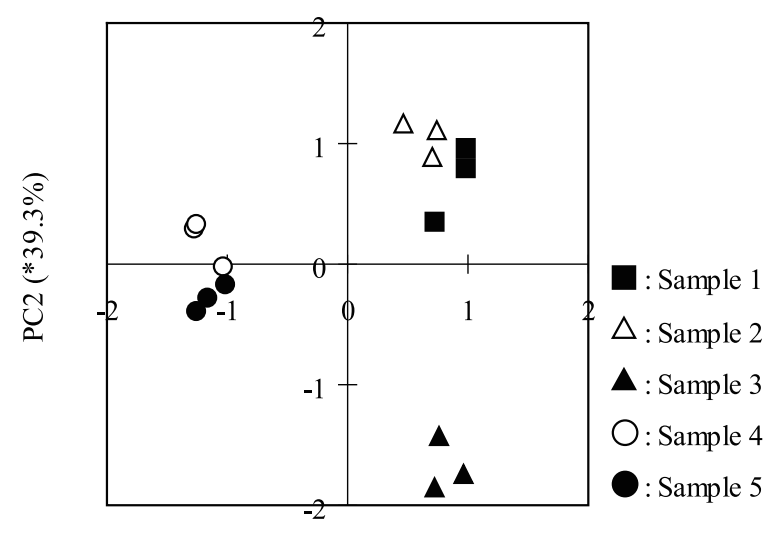

$\mathrm{PC} 1(* 43.4 \%)$

Fig. 3. Graphical representation of principal component analysis applied to the responses for milk and four types of reconstituted milk by odor sensor. *: proportion.

Table 4

Correlation coefficients among compounds, sensory attributes, and component scores applied to the responses by odor sensor

\begin{tabular}{lcr}
\hline & PC1 & \multicolumn{1}{c}{ PC2 } \\
\hline Milk flavor & 0.360 & 0.289 \\
Cooked flavor & 0.794 & 0.367 \\
\hline Hexanal & 0.786 & 0.589 \\
2-Heptanone & 1.000 & -0.005 \\
2-Nonanone & 0.979 & -0.199 \\
Hydrogen sulfide & 0.757 & 0.324 \\
Dimethy sulfide & 0.901 & 0.269 \\
\hline
\end{tabular}

strong "cooked flavor" and a large amount of volatile compounds, is located on the right side. The PC2 does not have any significance based on the results of this study.

\subsection{Taste sensor analysis}

The results of the taste sensor analysis are shown in Fig. 4, Table 5, Table 6, and Table 7. Table 5 shows the average electric potential and standard deviation in each channel of the taste sensor. The five types of milk were discriminated significantly by Ch.6 and Ch.7 using Tukey HSD test. Table 6 shows the results of examining the correlation among taste sensor, sensory evaluation, and volatile compounds. Channel 5 shows high correlation with the "cooked flavor." Figure 4 illustrates the results of PCA of the taste sensor response values. The proportion of the PC1 was 69\%, and the proportion of the PC2 was $18 \%$, totaling $87 \%$. The results can be substantially accounted for by this twodimensional diagram. The five types of milk, that is, the milk and the four types of reconstituted milk, were discriminated independently. 


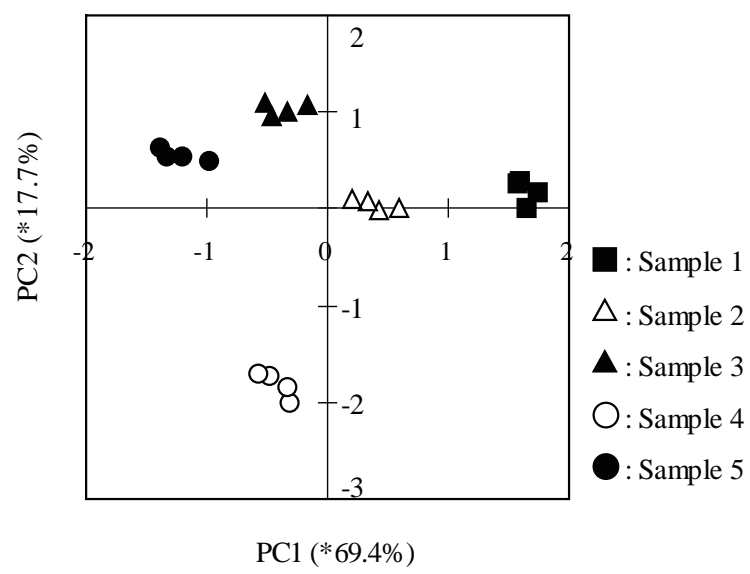

Fig. 4. Graphical representation of principal component analysis applied to the responses for milk and four types of reconstituted milk by taste sensor. *: proportion.

Table 5

Electric potential in each channel of the taste sensor.

\begin{tabular}{crccccccc}
\hline \multirow{2}{*}{ Sample No. } & \multicolumn{7}{c}{ Electric potential (mV) } \\
\cline { 2 - 8 } & Ch. 1 & Ch. 2 & Ch. 3 & Ch. 4 & Ch. 5 & Ch. 6 & Ch. 7 & Ch. 8 \\
\hline 1 & $0.87 \pm 0.11$ & $1.85 \pm 0.05$ & $-0.54 \pm 0.04$ & $0.55 \pm 0.10$ & $-0.85 \pm 0.06$ & $-1.19 \pm 0.09$ & $-0.64 \pm 0.08$ & $-0.61 \pm 0.19$ \\
2 & $0.32 \pm 0.11$ & $1.14 \pm 0.05$ & $-0.09 \pm 0.04$ & $0.05 \pm 0.08$ & $-1.20 \pm 0.14$ & $-2.35 \pm 0.02$ & $-1.36 \pm 0.06$ & $-2.21 \pm 0.19$ \\
3 & $0.27 \pm 0.15$ & $0.72 \pm 0.03$ & $-0.08 \pm 0.02$ & $0.25 \pm 0.09$ & $-1.60 \pm 0.12$ & $-3.15 \pm 0.02$ & $-3.52 \pm 0.04$ & $-3.64 \pm 0.28$ \\
4 & $0.71 \pm 0.15$ & $0.56 \pm 0.06$ & $-1.39 \pm 0.04$ & $-0.43 \pm 0.08$ & $-2.15 \pm 0.04$ & $-2.92 \pm 0.03$ & $-2.19 \pm 0.05$ & $-1.87 \pm 0.19$ \\
5 & $-0.05 \pm 0.14$ & $0.09 \pm 0.06$ & $-0.84 \pm 0.02$ & $-0.52 \pm 0.08$ & $-2.23 \pm 0.01$ & $-3.62 \pm 0.03$ & $-3.87 \pm 0.04$ & $-3.54 \pm 0.17$ \\
\hline
\end{tabular}

mean $\pm \operatorname{SD}(n=4)$

Table 6

Correlation coefficients among sensory attributes, compounds, and the response by the taste sensor.

\begin{tabular}{lrrrrrrrr}
\hline & \multicolumn{8}{c}{ Taste sensor } \\
\cline { 2 - 9 } & Ch. 1 & Ch. 2 & Ch. 3 & Ch. 4 & Ch. 5 & Ch. 6 & Ch. 7 & Ch. 8 \\
\hline Milk flavor & 0.347 & 0.325 & -0.389 & 0.213 & 0.330 & 0.252 & 0.467 & 0.246 \\
Cooked flavor & 0.523 & 0.783 & 0.188 & 0.721 & 0.817 & 0.671 & 0.739 & 0.509 \\
Sweetness & 0.323 & 0.537 & -0.084 & 0.350 & 0.584 & 0.486 & 0.682 & 0.414 \\
Saltiness & -0.502 & -0.076 & 0.333 & 0.342 & 0.194 & -0.270 & -0.382 & -0.623 \\
Milk taste & 0.257 & 0.245 & -0.443 & -0.061 & 0.227 & 0.257 & 0.563 & 0.361 \\
Viscosity & -0.086 & 0.105 & -0.250 & 0.121 & 0.246 & -0.010 & 0.187 & -0.120 \\
Fattiness & 0.199 & 0.138 & -0.537 & -0.122 & 0.128 & 0.133 & 0.447 & 0.243 \\
Chalkiness & 0.514 & 0.350 & -0.037 & 0.533 & 0.286 & 0.235 & 0.123 & 0.128 \\
Aftertaste & 0.205 & 0.098 & -0.591 & -0.143 & 0.080 & 0.088 & 0.403 & 0.210 \\
\hline Hexanal & 0.589 & 0.966 & 0.544 & 0.824 & 0.971 & 0.938 & 0.894 & 0.746 \\
2-Heptanone & 0.244 & 0.761 & 0.705 & 0.942 & 0.900 & 0.620 & 0.454 & 0.245 \\
2-Nonanone & 0.197 & 0.657 & 0.645 & 0.915 & 0.801 & 0.495 & 0.307 & 0.113 \\
Hydrogen sulfide & 0.555 & 0.923 & 0.787 & 0.895 & 0.910 & 0.903 & 0.703 & 0.674 \\
Dimethyl sulfide & 0.513 & 0.943 & 0.754 & 0.966 & 0.979 & 0.878 & 0.703 & 0.592 \\
\hline
\end{tabular}


Table 7

Correlation coefficients between sensory attributes and component scores applied to the responses by taste sensor.

\begin{tabular}{lrr}
\hline & PC1 & \multicolumn{1}{c}{ PC2 } \\
\hline Milk flavor & 0.298 & -0.372 \\
Cooked flavor & 0.751 & -0.015 \\
Sweetness & 0.517 & -0.212 \\
Saltiness & -0.149 & 0.732 \\
Milk taste & 0.246 & -0.528 \\
Viscosity & 0.060 & -0.040 \\
Chalkiness & 0.314 & -0.026 \\
Fattiness & 0.133 & -0.537 \\
Aftertaste & 0.091 & -0.563 \\
\hline Hexanal & 0.958 & 0.113 \\
2-Heptanone & 0.712 & 0.571 \\
2-Nonanone & 0.601 & 0.599 \\
Hydrogen sulfide & 0.920 & 0.331 \\
Dimethy sulfide & 0.923 & 0.382 \\
\hline
\end{tabular}

Table 7 shows the results of examining the correlation of these taste sensor analysis results with the sensory evaluation and volatile compounds. The results show high correlation of the PC1 in the taste sensor analysis with the "cooked flavor" and every volatile compound, particularly with hexanal, hydrogen sulfide, and dimethyl sulfide. On the basis of the previously reported finding that the contribution of those two types of sulfur compounds to "cooked flavor" is high, ${ }^{(6)}$ this suggests that the PC1 can be interpreted as an axis indicating the strength of "cooked flavor." On the PC1 axis, the direction of shifting from Sample 3 to Sample 2 and that from Sample 5 to Sample 4 were both positive directions. It was thought that the result that the raw milk contributed to the addition of richness caused by "cooked flavor" was objectively shown. On the other hand, the PC2 had a high correlation with "saltiness," suggesting that the PC2 can be interpreted as an axis indicating the intensity of "saltiness." This result corresponded to the evaluation that in "saltiness" Sample 4 was the most slight and Sample 5 the strongest out of the five samples. Despite the same blending ratios, such as Sample 3 and Sample 5, the evaluation of "saltiness" differed. It was considered that the flavor characteristic obtained by combining various types of milk materials and different pasteurization methods can be evaluated by "saltiness." In this experimental system, the taste sensor analysis provides results that support the sensory evaluation.

\section{Discussion}

As a new technique of flavor evaluation, we have studied the applicability of sensor analysis to the flavor of milk and reconstituted milk processed through the combination of various types of milk materials and pasteurization methods. Using sensory evaluation, we first defined the flavor characteristics of a total of five types of milk, including 
milk and four types of reconstituted milk. We found that, in order to add richness, it is effective to mix raw milk and conduct Plate-type pasteurization, and in order to give a plain flavor finish, it is effective to conduct Inf-type pasteurization. GC analysis revealed the trend that samples with a plain flavor did not have a sufficient level of detected volatile compounds. Organizing the results of the odor sensor and the taste sensor analyses in relation to the results of the sensory evaluation and GC analysis, with the odor sensor we found a correlation between the amount of volatile compounds and "cooked flavor," and with the taste sensor we found a correlation between "cooked flavor" and "saltiness." This suggests that discrimination can be made significant by combining the sensor analysis results with the flavor characteristics obtained in the sensory evaluation or GC analysis, and that they can be indicated objectively.

We therefore conclude that the sensor analysis used in this experimental system is an effective method for the objective evaluation of flavors. However, this experimental system was simple. It used common milk materials. In products on the market, the combination of milk materials is more complex. Therefore, when examining such combinations, it is necessary that the examination be performed in an experimental system that contains many other milk materials.

\section{References}

1 H. E. Nursten: Int. J. Dairy Technol. 50 (1997) 48.

2 K. Iwatsuki, Y. Mizota, M. Sumi, K. Sotoyama and M. Tomita: J. Jpn. Soc. Food Sci. Technol. 46 (1999) 535.

3 K. Iwatsuki, T. Konno, Y. Mizota, K. Sotoyama, M. Sumi and M. Tomita: J. Jpn. Soc. Food Sci. Technol. 47 (2000) 844.

4 K. Iwatsuki, H. Matsui, Y. Mizota, K. Sotoyama, M. Sumi and M. Tomita: J. Jpn. Soc. Food Sci. Technol. 48 (2001) 126.

5 K. Toko: Biomimetic Sensor Technology (Cambridge University Press, UK, 2000) Chap. 6.

6 Y. Mizota, H. Matsui, M. Ikeda, K. Iwatsuki and K. Toko: Jpn. J. Taste Smell Res. 13 (2006) 517.

7 Y. Mizota, H. Matsui, M. Ikeda, N. Ichihashi, K. Iwatsuki and K. Toko: Jpn. J. Taste Smell Res. 14 (2007) 471.

8 K. Toko, T. Iyota, Y. Mizota, T. Matsuno, T. Yoshioka, T. Doi, S. Iiyama, T. Kato, K. Yamafuji and R. Watanabe: Jpn. J. Appl. Phys. 34 (1995) 6287.

9 H. Yamada, Y. Mizota, K. Toko and T. Doi: Mater. Sci. Eng. C 5 (1997) 41. 